\title{
Development of an intelligent videogrammetric wind tunnel measurement system
}

\author{
Sharon S. Graves and Alpheus W. Burner \\ NASA Langley Research Center \\ Hampton, VA 23681-2199
}

\begin{abstract}
A videogrammetric technique developed at NASA Langley Research Center has been used at five NASA facilities at the Langley and Ames Research Centers for deformation measurements on a number of sting mounted and semispan models. These include high-speed research and transport models tested over a wide range of aerodynamic conditions including subsonic, transonic, and supersonic regimes. The technique, based on digital photogrammetry, has been used to measure model attitude, deformation, and sting bending. In addition, the technique has been used to study model injection rate effects and to calibrate and validate methods for predicting static aeroelastic deformations of wind tunnel models. An effort is currently underway to develop an intelligent videogrammetric measurement system that will be both useful and usable in large production wind tunnels while providing accurate data in a robust and timely manner. Designed to encode a higher degree of knowledge through computer vision, the system features advanced pattern recognition techniques to improve automated location and identification of targets placed on the wind tunnel model to be used for aerodynamic measurements such as attitude and deformation. This paper will describe the development and strategy of the new intelligent system that was used in a recent test at a large transonic wind tunnel.
\end{abstract}

Keywords: videogrammetry, photogrammetry, model deformation, model attitude, pattern recognition, computer vision, fuzzy image processing

\section{INTRODUCTION}

An effort is currently underway at NASA Langley to improve and optimize videogrammetric techniques suitable for robust and routine use in major NASA facilities for the measurement of model attitude and deformation. The measurement of model attitude is fundamental to wind tunnel testing. Accurate and reliable measurements are required for the computation of lift and drag from normal and axial forces. In addition, the accurate measurement of wind tunnel model deformation is essential in understanding the effects of control and lifting surfaces on aerodynamic performance. Performance differences caused by unknown control or lifting surface position or movement, e.g. model deformation, can decrease data confidence and cause discrepancies between measured values and those predicted based on rigid body assumptions.

Automatic image processing and close range photogrammetry are becoming standard tools for the measurement of wind tunnel model deformation and are widely used in industry to accurately model three-dimensional (3-D) structures. The development of videogrammetric model deformation (VMD) techniques with multiple cameras for use in wind tunnels dates back to the 1980s [1]. Advanced image processing coupled with the development of a simplified single-camera, single-view technique has permitted automated data acquisition and reduction facilitating near routine use in production wind tunnels [2]. As computers attain the processing speed required to emulate human intellectual capabilities, we turn our attention to the development of an intelligent videogrammetric measurement system capable of learning from experience, self-organizing its programming, and adjusting its course of action for standard use in production wind tunnels. Computer vision is used as a primary source of knowledge for monitoring model deformation as well as changing environmental and wind tunnel conditions. In addition to having tools that increase automation using precise, crisp rules of behavior or well-defined symbols usually extracted from human expertise, the system is designed to emulate a biological intelligence operating on data that are not well-defined but are "fuzzy" or subject to interpretation and have some degree of uncertainty [3].

Typically, an industrial-grade, video-rate, charged coupled device (CCD) camera and a frame grabber board are used to record image sequences of static or dynamic objects for analysis to extract useful information. Image acquisition and processing are provided by a distributed, parallel-processing architecture to enhance real-time capabilities. Advanced pattern recognition techniques are used to improve automated location and identification of targets used for aerodynamic 
measurements. Close range photogrammetry is then used to extract accurate two-dimensional (2-D) or 3-D spatial measurements that are then transformed into the engineering variables of interest (deformation, attitude, etc.) The use of visual input is maximized to automate calibration and initial test setup. Representative data are included to illustrate typical problems encountered by optical systems in a wind tunnel environment. This work is part of an overall effort to develop a robust and usable static and dynamic model deformation measurement capability (up to $1000 \mathrm{~Hz}$ ) useful for a wide variety of wind tunnel testing that may also have potential for the measurement of model attitude as a complement to inertial sensors. Emphasis is placed on the design of a highly parallel processing system rather than a computer architecture that processes data sequentially. [

This paper describes the development of a robust, highly accurate, "smart" videogrammetric measurement instrument using advanced non-traditional methods to make possible routine use in production wind tunnels. The system is being developed to encode a higher degree of knowledge, provide faster execution, be less prone to error, and require less maintenance.

\section{VIDEOGRAMMETRY}

The videogrammetric measurement technique is an optical method characterized by automated image processing, sub-pixel resolution, near routine, near real-time measurements, and high data volume with minimum impact to productivity [4]. The technique consists of a single-camera, single-view, photogrammetric solution from digital images of targets placed on the wing at known semi-span locations. Except for the targets, the technique is non-intrusive. When a light source is positioned near the camera the light retro-reflected from the tape targets can greatly exceed that possible with white diffuse targets, resulting in a high contrast image in which the targets are easily discriminated from the background. Such high contrast images are amenable to automated image processing. Polished paint targets with less contrast are used when target thickness is an issue.

Acquisition and digitization of a live video stream at a nominal $60 \mathrm{~Hz}$ (up to $320 \mathrm{~Hz}$ in some cases) can be triggered by the facility Data Acquisition System (DAS). Once the video sequence is acquired, a blob analysis is used for target detection in the image. A gray-scale centroid calculation with the background level automatically removed provides sub-pixel accuracy. Single-view photogrammetry is then used to determine the $X$ (stream-wise) and $Z$ (vertical) coordinates in object space, given the known $Y$ (cross-flow) coordinates. Z-intercept location and slope angles of the target row are computed by a linear least squares fit in $X-Z$ space for each semispan station along the wing [5].

\subsection{Primary Data Reduction}

The VMD measurement technique uses photogrammetry to extract two-dimensional (2D) images and map them into a three dimensional (3D) object space. The collinearity equations provide a mapping of coordinates between 3D object space and the 2D coordinates in the image plane [6], [7]. The nth target location point $p_{n}=\left(x_{n}, y_{n}\right)^{T}$, in the image plane is related to a point $P_{n}=\left(X_{n}, Y_{n}, Z_{n}\right)^{T}$, in the object space by

$$
\begin{aligned}
& x_{n}-x_{p}+d x=-c \frac{\cos \phi \cos \kappa\left(X_{n}-X_{c}\right)+(\sin \omega \sin \phi \cos \kappa+\cos \omega \sin \kappa)\left(Y_{n}-Y_{c}\right)+(-\cos \omega \sin \phi \cos \kappa+\sin \omega \sin \kappa)\left(Z_{n}-Z_{c}\right)}{\sin \phi\left(X_{n}-X_{c}\right)+(-\sin \omega \cos \phi)\left(Y_{n}-Y_{c}\right)+\cos \omega \cos \phi\left(Z_{n}-Z_{c}\right)} \\
& y_{n}-y_{p}+d y=-c \frac{-\cos \phi \sin \kappa\left(X_{n}-X_{c}\right)+(-\sin \omega \sin \phi \sin \kappa+\cos \omega \cos \kappa)\left(Y_{n}-Y_{c}\right)+(\cos \omega \sin \phi \sin \kappa+\sin \omega \cos \kappa)\left(Z_{n}-Z_{c}\right)}{\sin \phi\left(X_{n}-X_{c}\right)+(-\sin \omega \cos \phi)\left(Y_{n}-Y_{c}\right)+\cos \omega \cos \phi\left(Z_{n}-Z_{c}\right)}
\end{aligned}
$$

The interior orientation of the camera is given by the parameter set $\left(c, x_{p}, y_{p}\right)$. The exterior orientation of the camera is given by the parameter set $\left(\omega, \phi, \kappa, X_{c}, Y_{c}, Z_{c}\right)$, where $\omega, \phi, \kappa$ are rotational Euler angles and $X_{c}, Y_{c}, Z_{c}$ represent the coordinates of the perspective center of the camera in object space. The $d x$ and $d y$ terms are predominantly due to lens distortion.

In a single camera VMD system, a solution $(X, Y, Z)$ to the collinearity equations from a single set of image coordinates $(x, y)$ is not possible unless additional information is provided. For motion confined to the pitch plane, the spanwise locations $(Y)$ of the targets are fixed so that the number of unknowns reduces to 2, namely $X$ and $Z$. 


\section{SYSTEM}

\subsection{Experimental Setup}

A typical experimental setup for measuring model attitude in a wind tunnel facility is shown in Figure 1. The camera and (possibly) fiber optic lighting are positioned to view the model located in the test section of the wind tunnel. The field-ofview must be sufficient so as not to lose sight of the targets when the model is pitched. The videogrammetry acquisition system, consisting of a frame grabber and a Personal Computer (PC) located in a nearby control room can be interfaced to the main wind tunnel Data Acquisition System (DAS) via an Ethernet connection. The basic hardware consists of one or more instrumentation-grade analog or digital CCD cameras, a frame grabber board, and a Pentium III- or higher-based computer with custom image acquisition and reduction software. If retro-reflective targets are used, a fiber optic light source is positioned as close to the camera as possible to take advantage of the retroreflective nature of the optical targets. If the thickness of the retro-reflective targets $(0.10 \mathrm{~mm})$ is a concern then polished paint targets $(<10 \mu \mathrm{m})$ can be used. Existing test section lighting is typically used to illuminate the polished paint targets. The camera is usually positioned for an oblique view of the model at an angle of about 20 to $40^{\circ}$ to the horizontal for model deformation measurements. Targets are typically placed in rows at a number of known semispan locations, $\eta$, (or known locations relative to the control surface of interest) from near the wing root to near the wing tip. The diameters of the targets are selected to span at least 5 pixels on the image plane [4].

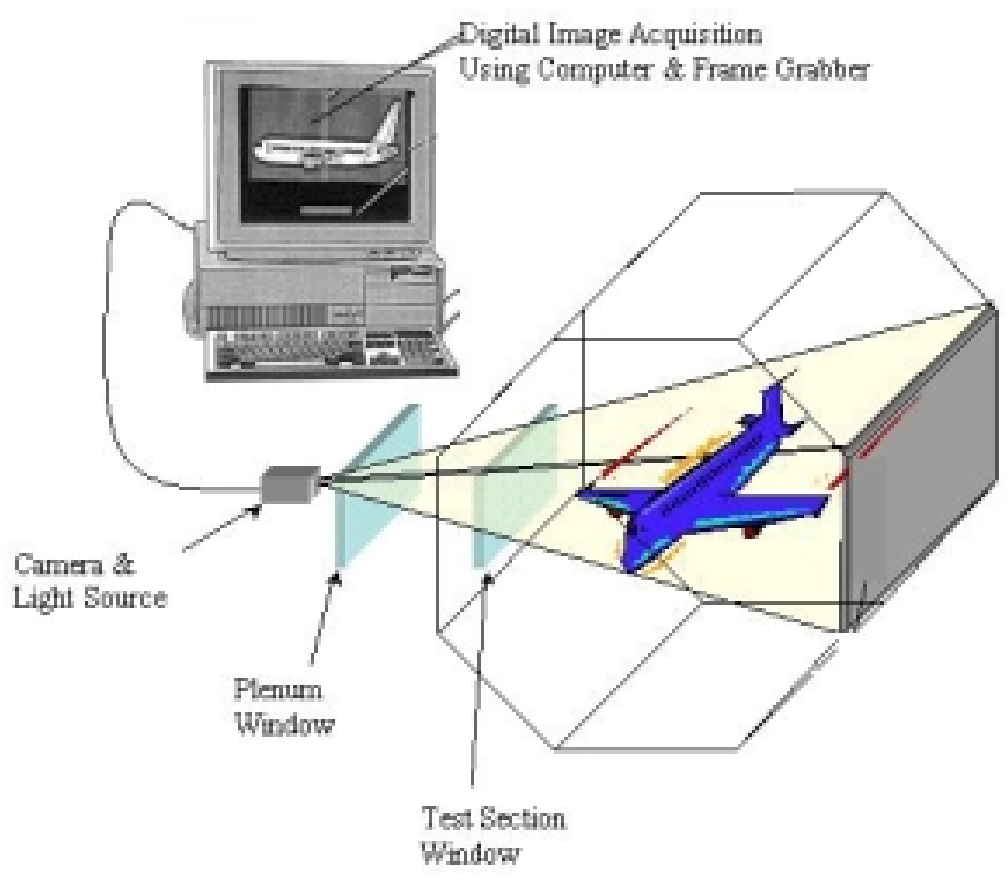

\subsection{Targets and Identification}

Figure 1: Typical experimental setup for a wind tunnel facility.

Targets on the model are used to establish a relation between 2-D coordinates in the image and 2-D or 3-D coordinates in object space. Targets are selected to provide adequate contrast, ranging from black on a light background to white on a dark background to retro-reflective tape targets, in order to accommodate a variety of conditions. Retro-reflective targets were used for the first major facility test of the intelligent system on the DARPA/Northrop Grumman Smart Wing Un-piloted Combat Air Vehicle (UCAV) recently tested at the NASA Langley Transonic Dynamics Tunnel. Targets were placed on the smart control surface, seen on left side of Figure 2, in order to measure the shape of the control surface at selected locations. A more detailed image of the targets on the flap is shown in Figure 3. The hingeless flap has 10 segments that were independently controlled to produce various flap shapes. Static and dynamic time histories (3-second records at $60 \mathrm{~Hz}$ ) of the shape of segments 3 and 6 were automatically acquired and reduced with the prototype intelligent videogrammetric measurement system. The system was used in an automated mode throughout the test for over 2000 data points without a major malfunction. 


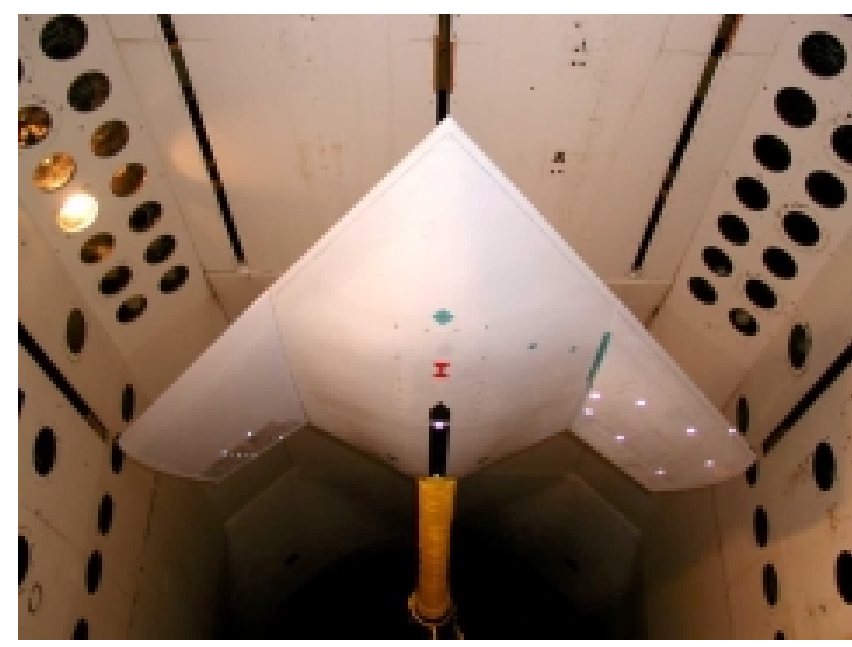

Figure 2: DARPA/AFRL/NASA/Northrop Grumman Smart Wing model as tested in NASA Langley TDT.

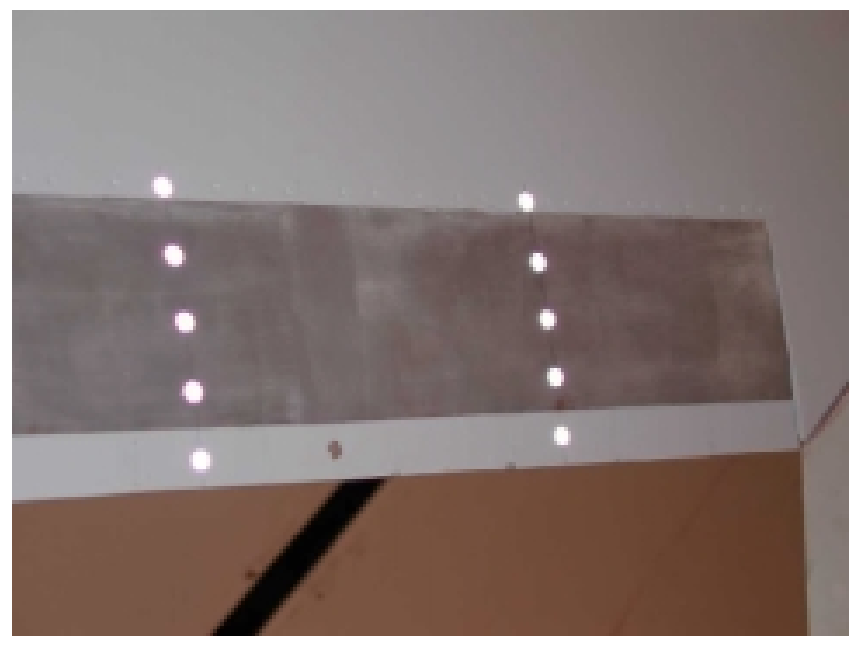

Figure 3: Retro-reflective targets on smart flap.

An example of polished painted targets is shown in figure 4. The model is a Boeing 777 under preparation for testing at the NASA Langley National Transonic Facility (NTF). Nine white targets can be clearly seen on the wing, a tenth target is just visible on the inboard portion of the wing and two targets on the upper left of the fuselage are not visible on the image. The contrast of the nine targets is much higher than the other three targets due to a black far-side wall that is seen as a specular reflection on most of the wing that tends to emphasize the white diffuse painted targets. For the three targets with low contrast the black wall is not seen in reflection and the contrast is very poor. This particular image was acquired with a digital still camera at a slightly different location than that used by the data camera and is presented to illustrate the difficulties that can arise with illumination.
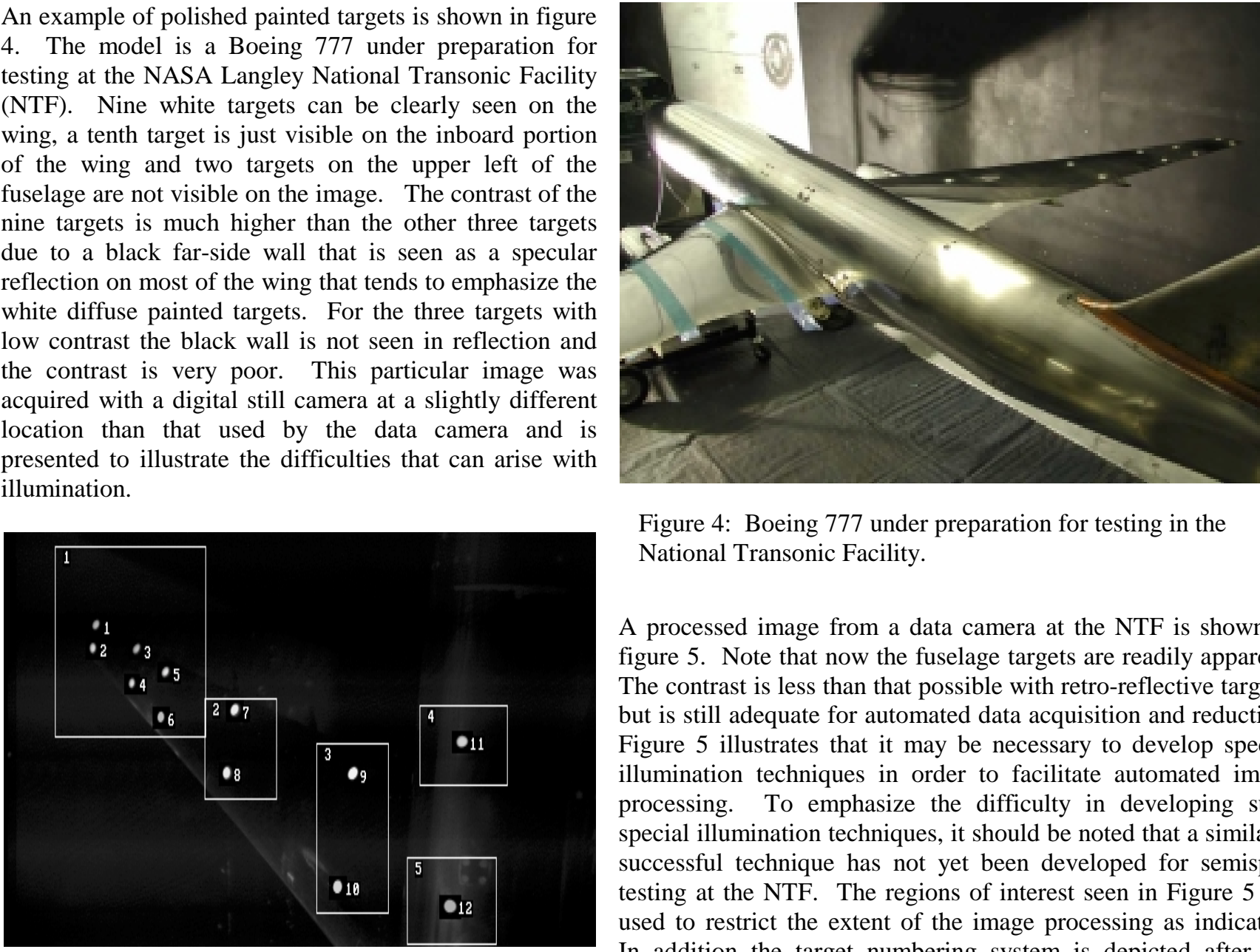

Figure 4: Boeing 777 under preparation for testing in the National Transonic Facility.

A processed image from a data camera at the NTF is shown in figure 5. Note that now the fuselage targets are readily apparent. The contrast is less than that possible with retro-reflective targets, but is still adequate for automated data acquisition and reduction. Figure 5 illustrates that it may be necessary to develop special illumination techniques in order to facilitate automated image processing. To emphasize the difficulty in developing such special illumination techniques, it should be noted that a similarly successful technique has not yet been developed for semispan testing at the NTF. The regions of interest seen in Figure 5 are used to restrict the extent of the image processing as indicated. In addition the target numbering system is depicted after the

Figure 5: Processed image from a data camera at the NTF for the model shown in Figure 4 showing regions of interest and target labels. measurement system has automatically located and identified the correct targets. 


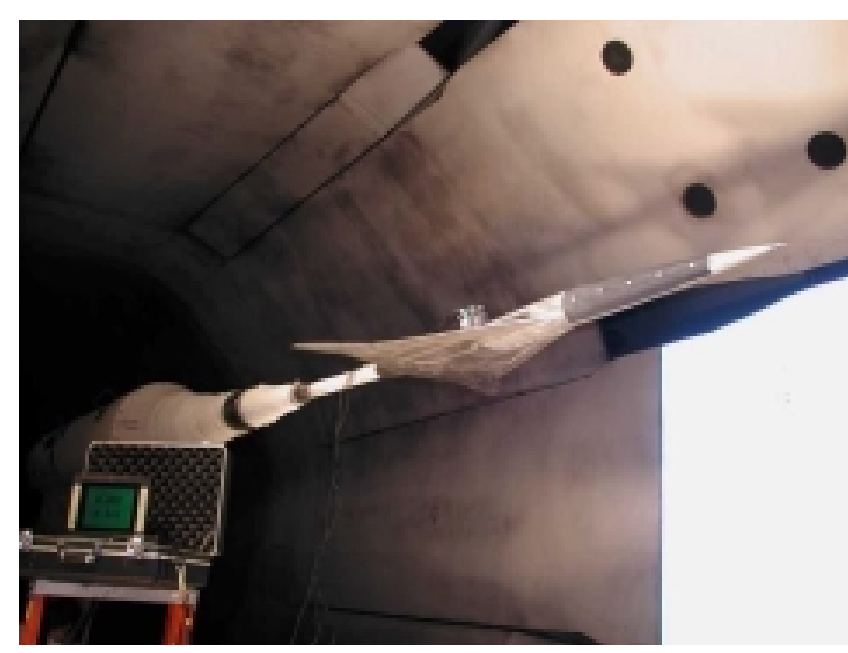

Figure 6: Check standard model at the NASA Langley 16-Ft Transonic Tunnel
In optical measurement systems, changing lighting conditions often affect the quality of the image. Titus [8] points out the critical nature of lighting for machine vision applications. Proper lighting can make the difference between success and failure for a vision system. Good imagery is a precursor to any successful machine vision measurement system, especially for

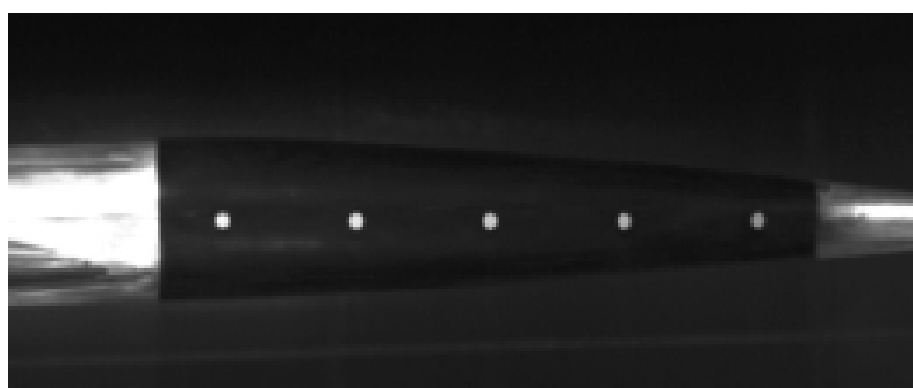

Figure 7: Image from a data camera of the model shown in figure 6 .

one that must operate in an automated manner with hands-off operation. Figure 6 illustrates another example of polished painted targets that were used, in this case, for the determination of model pitch angle. The model is a high-speed research model normally used as a check-standard model at the NASA Langley 16-Ft Transonic Tunnel for statistical quality control. Note that the retro-reflective screen (used for shadowgraph flow visualization) seen on the far-side wall to the right of the model makes it difficult to use retro-reflective targets at that facility since a very bright background results from on-axis illumination. Considerable off-axis illumination was required to obtain images (figure 7) of the polished paint targets with sufficient contrast in order to avoid the bright background that would result from nearly on-axis illumination. This example illustrates that adequate contrast for automated measurements is possible with a judicious selection of lighting location and the use of polished painted targets. Image processing is important for such applications, but is only one consideration of many regarding the complete system.

\subsection{Camera Calibration}

During camera calibration image plane correction parameters for lens distortion and lens alignment to the CCD sensor, as well as the determination of the location and pointing angles of the camera in the test section coordinate system, are determined. The parameters for image plane corrections are sometimes determined in a laboratory prior to setting up the measurement system in the test section.

A calibration fixture consisting of a number of targets with known spatial coordinates is used to determine lens distortion and principal distance, as well as to determine the orientation of the camera in the test section coordinate system. The calibration fixture is placed to fill the field-of-view of the camera for the distortion computations and is not necessarily aligned to the model for this phase of the calibration. Once the measurement system is set up with the proper view of the model, a calibration fixture is aligned to the test section coordinate system (Figure 8) in order to determine the pointing angles and location of the camera via photogrammetric space resection.

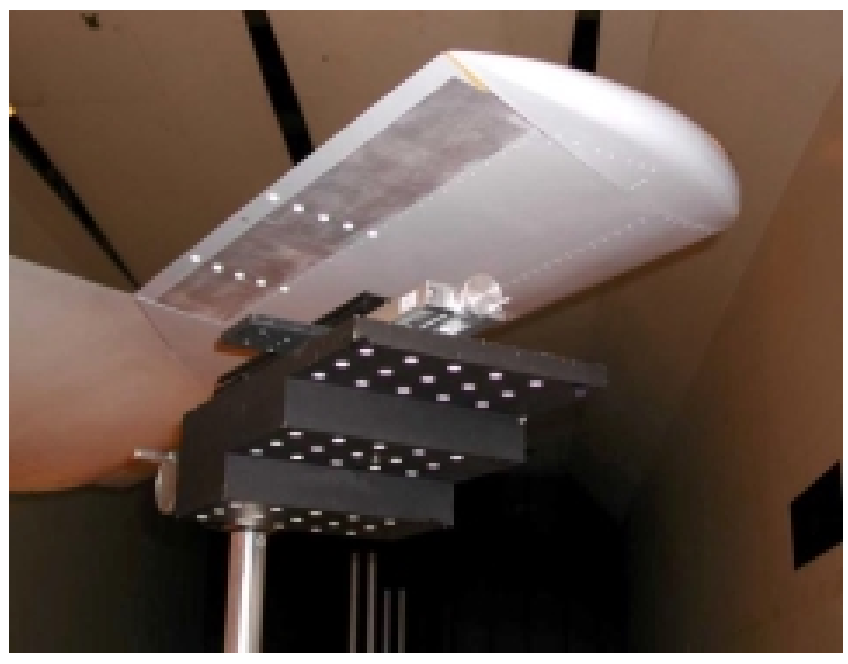

Figure 8: Calibration plate underneath model at the Langley Transonic Dynamics Tunnel for Smart Wing Test shown in Figures 2and 3.

\subsection{Automated target tracking}

Operations of routine tasks performed by the facility operator can be automated through a variety of image processing techniques. Introducing automatic thresholding of targets, target detection algorithms and greater flexibility in pattern 
recognition let the system handle a wider variety of tasks. Data fusion of multiple data acquisition systems through networked operations increases data throughput and data quality An intelligent measurement system must be able to properly handle both high contrast and low contrast targets that may be present in the same image.

\section{INTELLIGENT MEASUREMENTS}

A computer vision approach is used to acquire, process, analyze and understand higher dimensional data from 2-D images allowing the system to gain knowledge about the model, its size and configuration as well as the wind tunnel test environment. This ability to gain knowledge and learn from experience is a key ingredient in our definition of an intelligent system, laying the groundwork for self-organizing its algorithms and adjusting its course of action for standard use in production wind tunnels. The optical information processing for the computer vision task comprises many interacting levels. At the lowest level, the image is acquired and initial segmentation is used to delineate targets and patterns of interest. Image processing at this level, for example, deals with pattern recognition of the same features of the model rotated at various pitch angles. At the intermediate level, processing provides a direct correspondence between points in the image plane and objects in the 3-D plane. The three dimensional data processing uses techniques based on videogrammetry and computational geometry to reconstruct the 3-D motion of the model. At the highest level of processing, features extracted in the lower levels are used to classify and recognize objects [9]. Neural networks, fuzzy logic, and machine reasoning (induction and analogy) are some of the processing techniques that can be used for these complex tasks [10].

Computer vision improves the speed and accuracy of measurements by offering autonomous algorithms that can replace facility operators and researchers in performing visual tasks during the wind tunnel test. When it is not possible to entirely replace human with autonomous algorithms, the goal becomes to make certain types of operations more efficient allowing visual tasks to be performed more reliably, more quickly and more accurately. The continuous surveillance of the aeroelastic model during test assures constant flow of accurate data. Lighting conditions may change, the model itself may be rotated and shifted in the test section due to sting bending and the system must adapt in response to visual input regarding the environment. Just as human vision uses the eye and a brain, sensing and processing of optical information in computer vision are performed by the cameras and the host computer.

\subsection{Pattern detection}

The intelligent videogrammetric measurement system under development and currently being used for initial testing utilizes an interconnected set of computer vision algorithms for pattern detection that are applicable when using static cameras, target tracking and explicit models. The system uses a flexible framework encompassing three basic methods for pattern detection and recognition:

(1) an image invariance method that uses a set of image pattern relationships to uniquely determine patterns of models being searched for [11];

(2) a model-based method where a model is defined for the object of interest and the system attempts to match this model to different parts of the image in order to find a fit [12];

(3) an example-based learning method that breaks an object down into components that can be located and then combined if their configuration is valid [13], [14].

A built-in flexibility is desired, allowing the operator/system to utilize the pattern-matching technique most appropriate to the site. Sometimes the most successful technique comprises several pattern-matching algorithms used together. Although images are represented and manipulated as a set of pixels, for near real-time operation it is usually more appropriate to perform pattern matching on centroid coordinates of an ensemble of possible targets. The targets are placed in rows that are identified as components of an object. A component-based approach to object detection is then used to search for the model by looking for its identifying components rather than the whole object. The approach consists of two parts: target detectors and a means to integrate components (or targets) in an image and determine whether the object is valid. A component-based framework takes advantage of known geometric properties of the model and addresses the issue of detecting objects that are partially occluded or show poor contrast with the background such as figure 9.

An image sequence of retro-reflective targets taken on a Micro Aerial Vehicle (MAV) at 867 frames/sec at the NASA Langley BART facility is shown in figure 9. Retro-reflective targets near the trailing edge of the MAV wing are seen to disappear and then reappear, not because the targets are moving in and out of the field-of-view of the camera or being blocked, but rather because the angle of the targets changes enough due to the dynamic motion of the MAV that the light return fluctuates drastically over short periods of time. Targets that disappear and then reappear are especially troublesome to techniques striving for full hands-off automation. 
Vision processing often requires handling of data and knowledge that is vague and uncertain when performing routine recognition and quantitative interpretation of objects in images. Optical pattern recognition provides a useful tool for target recognition and extraction, target tracking and machine vision. The incoming images are projections of the aerodynamic model in a wind tunnel test section and these are often characterized by fuzziness or grayness. The human visual system routinely handles uncertain information efficiently; however, this is a difficult task for the computer vision system. Geometric features can be subjected to a wide range of variations and the system must not only discern these features but characterize the object of interest as well. The wide-ranging parameters of the preprocessing stage must now be adapted to the particulars of the wind tunnel environment. As incoming data are collected, processed and analyzed, the vision system gains knowledge about the scene. Suitable features are extracted for measuring salient object properties and these are used to refine classification or object recognition parameters. The visual observations become an integral part of the vision system when the vision system actively responds (or adapts) to these observations.

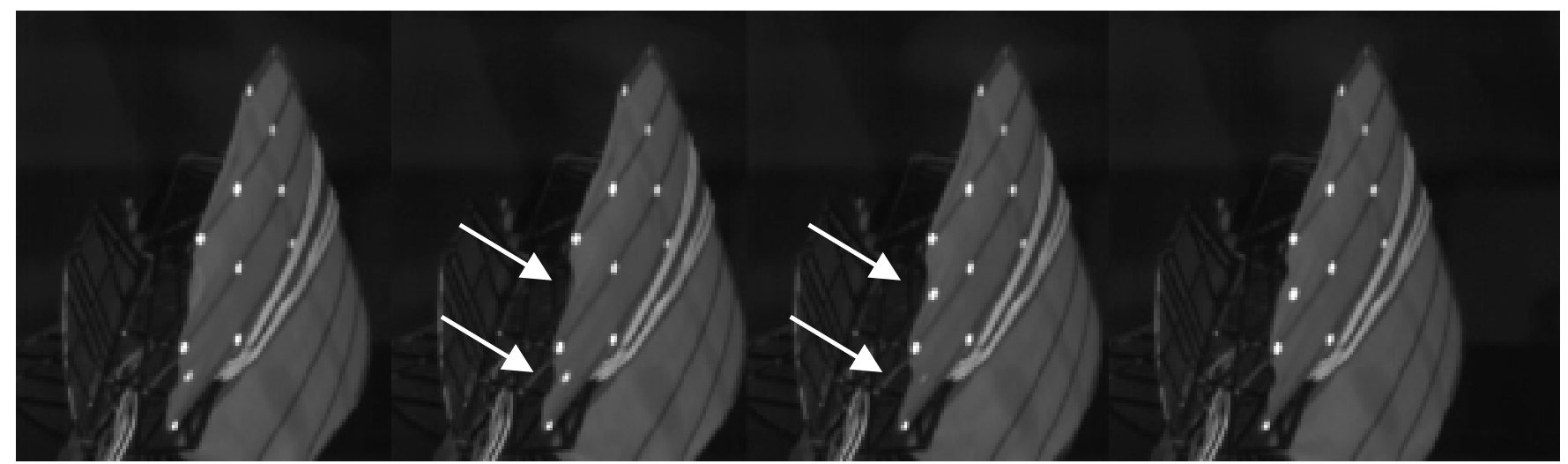

Figure 9: Micro Aerial Vehicle undergoing testing at the NASA Langley BART facility; an image sequence of retroreflective targets on a MAV taken at 867 frames/sec.

\subsection{Preprocess stage}

In the preprocess stage, wide-ranging search parameters are used to initially select the uncertainty levels, positional accuracy, speed parameters and search regions of the visual input. As soon as the system becomes operational, it uses data acquired during the wind off test points to refine search parameters such as the selection of optimized search regions and the range of angles to search. The preprocess stage uses knowledge gained about the model to decide on the optimal strategy for subsequent search operations. Providing a typical target image helps improve the search's robustness and optimize the strategy for subsequent search operations.

\subsection{Blob analysis}

Images are represented as sets of pixels, artifacts of the sensing device, having no relation to image content nor to our perception of image content. Image processing at this level is time-consuming and a blob analysis is often performed as an elimination process whereby only connected regions of pixels are considered in further analysis. A blob is an area of touching pixels that are in the same logical pixel state. Often blobs are used in feature extraction by selecting features that satisfy certain criteria. The raw data is reduced to a few measurements generally producing a more comprehensible and useful result.

\subsection{Fuzzy image processing}

Figure 10 illustrates troublesome glints that can arise from specular reflections due to components such as nacelles. Glints are a common source of ambiguity when processing images of a model in a wind tunnel test section. Glints are especially difficult to correctly identify because of their similarity in size and intensity to the targets, resulting in their incorrect classification using algorithmic methods. If the glints are mistakenly found as targets then the image coordinate data will be corrupted.

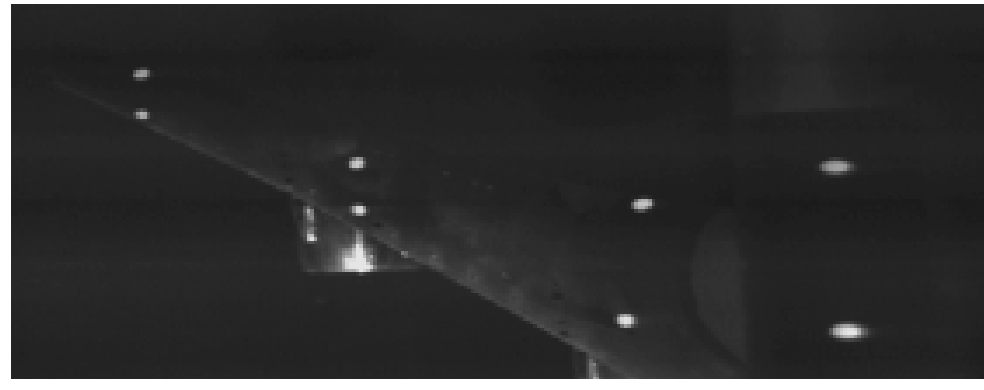

Figure 10: Model image showing troublesome glint from a nacelle. 
Fuzzy logic is a logic that deals with the uncertainty or vagueness inherent in how an object or event is characterized [15]. Fuzzy image processing provides a tool for making inferences from imperfect and incomplete data. Crisp rules allow elements of a set to have a membership of zero or one. Elements either belong to the set or they do not. Parameters relating the interconnectedness of pixels used to search for targets (or blobs) in an image are defined using these crisp rules allowing for searches based on brightness, blob area and size of interconnectedness often resulting in the incorrect classification of glints as blobs. Fuzzy sets extend this membership classification by introducing a degree of membership that ranges from 0 (not in the set) to one (definitely in the set) inclusive. Mathematically, the degree of membership is defined by a membership function, $\mu_{\mathrm{A}}(\mathrm{x})$ :

$$
A=\left\{\left(x, \mu_{A}(x)\right) \mid x \in X\right\} \text { where } \mu_{A}(x): X \rightarrow[0,1]
$$

Images as fuzzy sets can be represented by individual elements, $\mu_{\mathrm{A}}\left(\mathrm{x}_{\mathrm{i}}\right) / \mathrm{x}_{\mathrm{i}}$ called fuzzy singletons [16]. An image A of size M x N pixels with L gray levels can be defined as an array of fuzzy singletons (fuzzy sets with only one supporting point). For this application, the membership value $\mu_{\mathrm{A}}\left(\mathrm{x}_{\mathrm{mn}}\right)$ is designated by a predefined image property (brightness). The array is denoted as:

$$
A=\bigcup_{m=1}^{M} \bigcup_{n=1}^{N} \frac{\mu_{m n}}{x_{m n}}
$$

\subsubsection{Fuzzy set operations}

For two fuzzy sets, $\mathrm{A}$ and $\mathrm{B}$, the following pointwise operations are defined for all $x \in X$ :

$$
\begin{array}{ll}
\text { equality: } & A=B \Leftrightarrow \mu_{A}(x)=\mu_{B}(x) \\
\text { containment: } & A \subset B \Leftrightarrow \mu_{A}(x) \leq \mu_{B}(x) \\
\text { union: } & A \cup B, \mu_{A \cup B}(x)=\max \left\{\mu_{A}(x), \mu_{B}(x)\right\} \\
\text { intersection: } & A \cap B, \mu_{A \cap B}(x)=\min \left\{\mu_{A}(x), \mu_{B}(x)\right\} \\
\text { complement: } & \bar{A}, \mu_{A}(x)=1-\mu_{A}(x)
\end{array}
$$

The operation of fuzzification transforms a nonfuzzy set into a fuzzy set or increases the fuzziness of a fuzzy set.

\subsection{Model-based method}

In the model-based approach, a model is defined for the object of interest and the system attempts to match this model to the image in order to find a pattern match. Two types of errors in this type of pattern matching are predominant: (1) to miss a match or to not match a feature in an image and (2) to find a false match. Work has been devoted to estimation based on robust methods - ideally, even if a false match is found, a meaningful result can still be obtained. Solutions must be robust enough to handle variation introduced by sensitivity to noise, correlators highly sensitive to even small changes in input pattern, low contrast targets, limited illumination options, and limited view ports. Often an image must be normalized prior to object detection.

Normalized grayscale correlation. A suitable similarity measure of the model, $\mathrm{M}$ and image, I, where $\mathrm{M}$ and I are images of size $\mathrm{N}$ pixels, is given by the normalized cross-correlation function

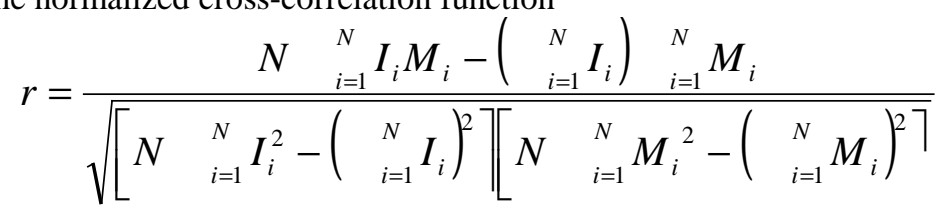

Result, $r$, reaches its maximum value of 1 where the model and the image match exactly. A zero result indicates the model and image are uncorrelated, and a negative value where the similarity is less than might be expected by chance. 
To speed up the search all terms in the normalized correlation that are associated only with the model are evaluated once when the model is defined. Only three terms need to be calculated during the search, $I, \quad I^{2}, \quad I M$.

This amounts to two multiplications and three additions for each model pixel. For a typical $128 \times 128$ pixel model in a $512 \times 512$ pixel image the total number of multiplications needed for this search is $2 \times 512^{2} \times 128^{2}$ an exhaustive search that would take too long. To reduce the number of computations, a hierarchical search utilizes a series of smaller, lower resolution versions of both the image and the model. The search is performed on a much reduced scale as seen in Figure 11 and proceeds to higher and higher resolutions to ensure that the matches found at lower resolution actually were occurrences of the model.

\subsection{Example based learning method}

In the learning method, the characterization of the aeroelastic deformation of the wing structure is performed in stages. Pattern recognition algorithms are required to be robust and fast since they are performed as the image is acquired. This is accomplished through a set of built-in pattern analysis tools. A pattern analysis function is performed with a two-dimensional reconstruction of the image. Movement of the pattern is defined in a sequence of static poses of the model through various patterns of movement. In the first stage, the target pattern is analyzed as the model is pitched in a wind-off condition. The pattern is tracked as a single "blob-like" entity through a range of pitch angles. Recognition of movement is accomplished through use of a temporal template [10], [17]. A
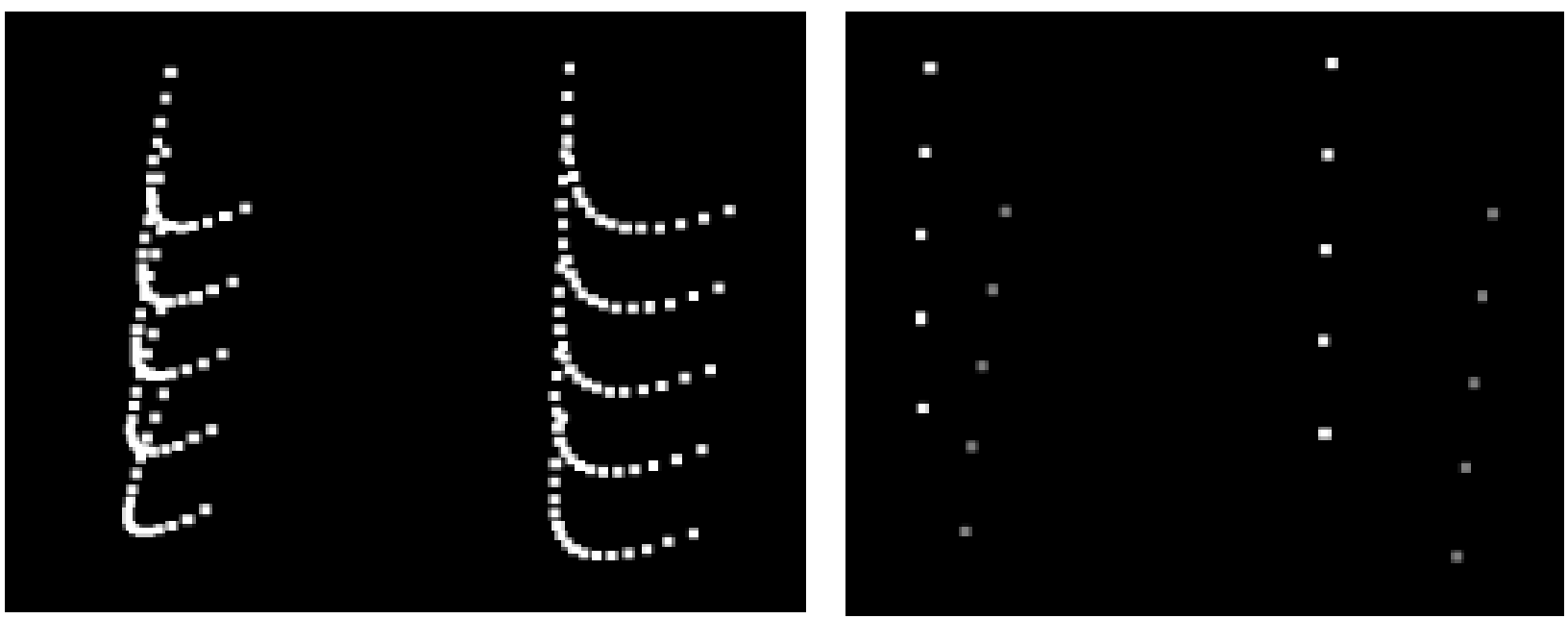

Figure 12: Overlapped images of the target motion on the Smart Wing flap as (a) the model is pitched showing a motion history for all angles and (b) an overlay showing the target pattern rotation at two pitch angles.

single image is used to record and display cumulative binary overall motion of the model. Multiple binary images are stored at different pitch angles. In Figure 12 a binary image depicts the blob-like entity representing the overlapping pattern of the target motion on the Smart Wing flap. The motion of the 10 targets on the flap is shown as the model is both pitched and the flap deflected.

Once the full range of motion of the pattern has been defined, the overall shape is used to hypothesize a fuzzy region of interest for robust and speedy recognition of target patterns. Movement of the wing structure viewed with a static background is defined as motion over time. The basic idea is to construct a temporal template defining the extent of movement encompassing specific representation of the movement (e.g., changes in pitch or flap angle) as well as predefined fuzzy regions using motion instead of structural features [17]. Key elements are identified as fuzzy sets in which the 
transition from membership to non-membership is gradual than abrupt. The acquired image is matched against the template having stored representations of known movements.

The image processing input data, consisting of gray levels is processed in the membership plane where the inference engine is used to make decisions, classify or aggregate data according to the fuzzy set rules. Prior to this, the image may go through an initial fuzzification where problem areas (such as brightness ambiguity, vagueness or ill-defined data) are assigned membership values with respect to the properties prior to reaching the membership plane.

\subsection{Fuzzy Logic and Machine Reasoning}

The brain of the system is a fuzzy inference engine containing the knowledge about the relations between the individual fuzzy input images and output data. The fuzzy inference engine comprises if-then conditions according to Equations 4. Machine reasoning techniques are applied to make the computer program evaluate a proposition and decide whether or not some action should be the consequent of the proposition. The proposition expressed in either Boolean or fuzzy logic (• represents a dyadic operator AND or OR) is a collection of predicates, $\mathrm{P}_{\mathrm{i}}$ that are evaluated before a set of actions, $\mathrm{A}_{\mathrm{i}}$ are taken. For example,

If $\mathrm{P}_{1} \bullet \mathrm{P}_{2} \bullet \mathrm{P}_{3} \ldots \mathrm{P}_{\mathrm{n}}$ then $\mathrm{A}_{1} \ldots \mathrm{A}_{\mathrm{n}}$

The reasoning system isolates and constructs the necessary relationships to prove the truth of the predicate proposition, and if the predicate is true, then a final classification of targets by their features is performed.

\section{SAMPLE WING TWIST AND DEFORMATION RESULTS}

Typical dynamic deformation contours for the first five images in a sequence are plotted in Figure 13 [5]. Video photogrammetry was used to measure dynamic deformation on the Models for A eroelastic Validation Research Involving Computation semispan model (MAVRIC-I), a business jet wing-fuselage flutter model, in NASA Langley's Transonic Dynamics Tunnel (TDT).

Figure 14 illustrates dynamic wing twist measurements taken on the same semispan model in the Transonic Dynamics Tunnel. The frequency of the variation of $\Delta Z$ and corresponding $\Delta \theta$ versus time of the five target rows for a typical data point near the flutter boundary for an aeroelastic semispan model are plotted. The frequency spectra were calculated by Fast Fourier Transforms (FFT) of the 5 second records of each $\Delta \theta$ and $\Delta \mathrm{Z}$ time history.

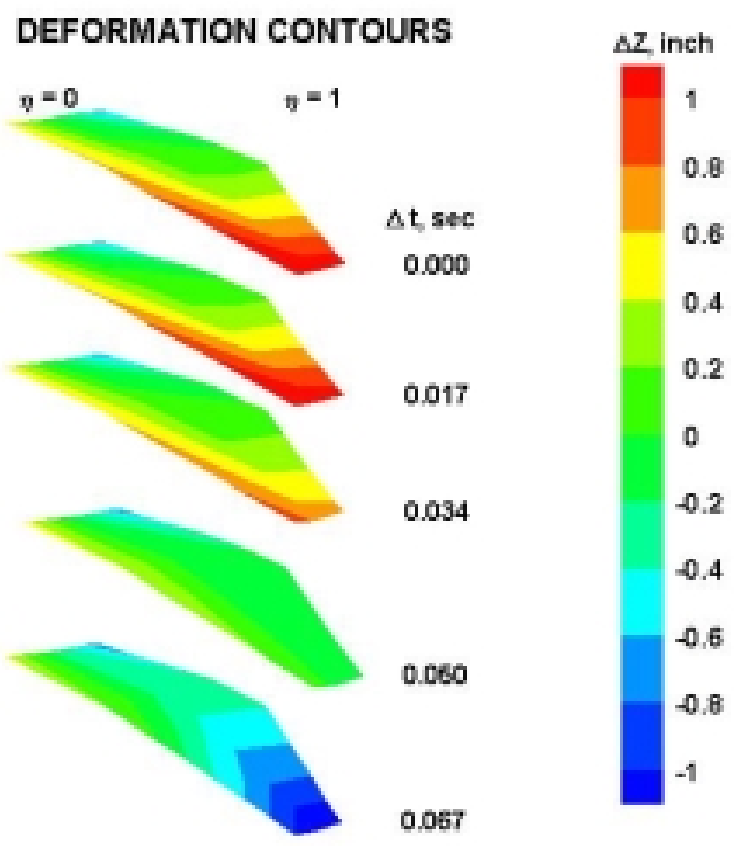

Figure 13: Deformation contours for the first five images in a sequence. 

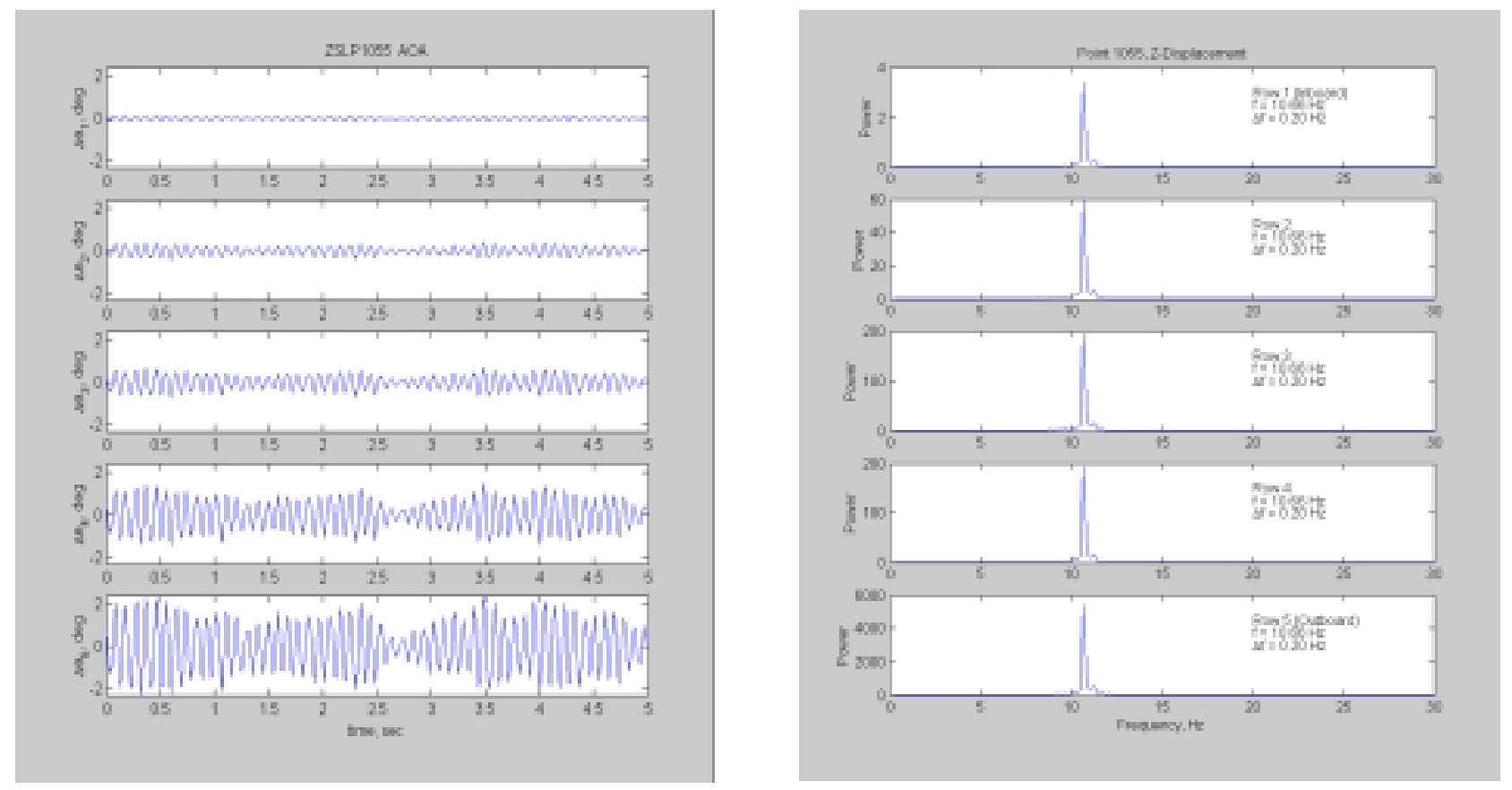

Figure 14: Delta wing twist, $\Delta \theta$, versus time and corresponding frequencies for 5 rows of targets.

\section{CONCLUDING REMARKS}

This paper highlights some valuable first steps in the development of an intelligent videogrammetric measurement system suitable for robust, routine use in production wind tunnels. Initial successes with a prototype system are encouraging. The prototype system performed well at a large transonic wind tunnel where it was used to measure static and dynamic shapes of 2 segments of a 10-segmented smart flap. The system was used in an automated mode throughout the test for over 2000 data points without a major malfunction. Enhancements under development include pattern matching to further increase the robustness of the system. Future work includes the use of templates created from existing features on the model as a nonintrusive replacement for the currently used retro-reflective targets. Modern image processing techniques will identify highly accurate pattern matches producing quality results with little labor. The complexity of target recognition and tracking capability is handled by the intelligence and adaptiveness of a knowledge based system. Reasoning and adaptive capabilities are built into the system through use of fuzzy logic allowing the system to automatically configure and train itself, reducing the need for "expert" operators.

\section{ACKNOWLEDGMENTS}

Part of the research described in this paper was conducted within the National Transonic Facility and Transonic Dynamic Facility at NASA Langley Research Center. The authors would like to acknowledge DARPA and Northrop Grumman for use of Smart Wing Test contributions. Donald Harper, Swales Aerospace, Inc. is acknowledged for his many contributions to the intelligent VMD systems under development for the TDT and NTF wind tunnels at NASA Langley Research Center. Harriett Dismond and Kenneth Cate are acknowledged for continuing support in VMD installations, calibration, and operations. The operations and research staff at the NASA Langley NTF and TDT are acknowledged for assistance and support in test technique developments and applications at their facilities. 


\section{REFERENCES}

1. A. W. Burner, W. L. Snow, and W. K. Goad, "Model Deformation Measurements at a Cryogenic Wind Tunnel Using Photogrammetry," $31^{\text {st }}$ ISA Symposium, San Diego, CA, May 1985; published in Instrumentation in the Aerospace Industry, vol. 31, ISA pp.615-622.

2. A. W. Burner and S. D. Martinson, "Automated Wing Twist Measurements under Aerodynamic Load," AIAA Paper 96-2253, June, 1996.

3. D. B. Fogel, T. Fukuda, and G. Ling, "Scanning the Issue/Technology: Special Issue on Computational Intelligence," Proceedings of the IEEE, Vol. 87, No. 9, September 1999.

4. A. W. Burner and T. Liu, "Videogrammetric Model Deformation Measurement Technique," Journal of Aircraft, vol. 38, no. 3, May-June 2001.

5. S. S. Graves, A. W. Burner, J. W. Edwards, D. M. Schuster, "Dynamic Deformation Measurements of an Aeroelastic semispan model", AIAA Paper 2001-2454, June, 2001.

6. K. W. Wong, "Basic Mathematics of Photogrammetry," Chapter 2, Manual of Photogrammetry, $4^{\text {th }}$ Edition, American Society of Photogrammetry, Va, p. 37-101, 1980.

7. J. C. McGlone, "Analytic Data-reduction Schemes in Non-topographic Photogrammetry," Chapter 4, NonTopographic Photogrammetry, $2^{\text {nd }}$ Edition, American Society for Photogrammetry and Remote Sensing, Va, p. 37$55,1989$.

8. Jon Titus, "Lights Make Machine Vision Shine," Test \& Measurement World, June, 2001.

9. T. Liu, S. S. Graves, R. W. Ross, K. B. Eom, J. M. Hereford, Computer Vision as a Universal Framework for Image-Based Measurements in Aerospace Engineering and Sciences, NASA Report for FY2000 ISE Project.

10. J. H. Elder, and R. M. Goldberg, "Image Editing in the Contour Domain," IEEE Transactions on Pattern Analysis and Machine Intelligence, vol. 23, no. 3, March, 2001.

11. P. Sinha, "Object Recognition via Image Invariants: A Case Study," Investigative Ophthalmology and Visual Science, vol. 35, p. 1735-40, May 1994.

12. A. Yuille, "Deformable Templates for Face Recognition," J. Cognitive Neuroscience, vol. 3, no. 1, PP 59=70, 1991.

13. A. Mohan, C. Papageorgiou, T. Poggio, "Example-based object detection in Images by Components," IEEE Transactions on Pattern Analysis and Machine Intelligence, Vol. 23, No. 4, April, 2001.

14. K. K. Sung, and T. Poggio, "Example-based learning for View-Based Human Face Detection," IEEE Trans. Pattern Analysis and Machine Intelligence, vol. 20, no.1, pp 39-51, Jan. 1998.

15. L. A. Zadeh, Fuzzy Sets. Information and Control, 8:338-353,1965.

16. H. HauBecker, and H. R. Tizhoosh, "Fuzzy Image Processing," Computer Vision and Applications, 20001, p 541571.

17. A. F. Bobick, and J. W. Davis, "The Recognition of Human Movement Using Temporal Templates," IEEE Transactions on Pattern Analysis and Machine Intelligence, vol. 23, no. 3, March 2001. 